\title{
EXPERIENCIA DE WEBINAR PARA MEJORAR LAS TUTORÍAS VIRTUALES EN LA ESPECIALIZACIÓN DE GESTIÓN DEL E-LEARNING Y DOCENCIA
}

\section{VIRTUAL WEBINAR EXPERIENCE TO IMPROVE VIRTUAL TUTORIES IN THE SPECIALIZATION OF E-LEARNING MANAGEMENT AND VIRTUAL TEACHING}

\author{
Carmen Graciela Arbulú Pérez Vargas ${ }^{1}$
}

\author{
Recibido: 2019-01-13 / Revisado: 2019-03-25 / Aceptado: 2019-04-01 / Publicado: 2019-07-01
}

Forma sugerida de citar: Arbulú-Pérez-Vargas, C. G. (2019). Experiencia de webinar para mejorar las tutorías virtuales en la especialización de gestión de e-learning y docencia. Retos de la Ciencia, 3(7), 74-84. https://doi.orq/10.53877/rc.3.7.20190701.07

\section{RESUMEN}

El presente trabajo tiene como objetivo analizar, las acciones tutoriales en la modalidad virtual, de estudiantes de la especialización de Gestión de e-Learning y Docencia Virtual de la Unidad de Estudios de Segunda especialidad de la Facultad de educación de la Universidad Nacional Pedro Ruíz Gallo de Lambayeque, Perú. Para ello se toma como base la teoría de Kurl Lewin sobre comunicación en grupo, así como la incorporación de elementos propuestos por Aristóteles como el argumentador, el tema, y el oyente, identificados como alumno, profesor, contenidos. Se diseña entonces, un plan de tutoría, basado en la aplicación del webinar como soporte tecnológico y con énfasis en un discurso del asesoramiento empático para conectar a los estudiantes y lograr los objetivos de aprendizaje en experiencias curriculares.

Palabras claves: tutoría, webinar, tecnología, asesoría.

\section{ABSTRACT}

The main objective of the study was to analyze the virtual mode tutorial activities of students of E- learning Management and Virtual Teaching in the School of Education, Unit of Second Major (Specialization), at Universidad National Pedro Ruiz Gallo, Perú. Kurt Lewin's theory of group communication was assumed as a basis, and elements proposed by Aristotle, as the arguer, the topic and the listener, identified as the student, the teacher, the contents. A Tutorial plan was then designed, on the basis of the application of webinar as a technological support and with emphasis on an empathic counselling to get connection with the students and achieve the learning objectives in curricular experiences.

Key words: tutorial, webinar, technology, counselling.

\footnotetext{
1 Profesora de la Escuela de Posgrado de la Universidad César Vallejo. Perú. Candidata a PhD por la Universidad Santo Tomás. Colombia. E-mail: carbulu@cvvirtual.edu.pe
} 


\section{INTRODUCCIÓN}

Nos encontramos en contextos de profunda digitalización de la sociedad, donde los límites y diferencias de profesiones y actividades, se hace menos evidente, con surgimiento de nuevas profesiones, lo cual exige una reestructuración profunda en el sistema educativo, como una manera de afrontar el avance tecnológico y la automatización para valorar y aprovechar las oportunidades y minimizar los problemas que estas demanden. (Comisión Europea, 2017)

Cabe precisar que el proceso de e-Learnigo aprendizaje electrónico está estrechamente vinculado a la gestión del conocimiento, rompe barreras geográficas, y en esta experiencia atendió a estudiantes de diversas regiones de Perú, en horarios flexibles, en la modalidad sincrónica y asincrónica.

Los entornos virtuales de aprendizaje han revolucionado la educación, adaptando el aprendizaje a las características, ritmo y estilos del estudiante; además facilitan la implementación de modelos pedagógicos colaborativos y constructivista pasando de la transmisión pasiva del conocimiento a la construcción de saberes. (Almeida, Silva y Santoyo, 2018)

Históricamente ideas como las de Aristóteles, que hacía referencia al orador, a quien se le atribuía la capacidad de emplear un discurso y persuadir al auditorio, tenía que ver qué con lo que dice, pero además cómo lo dice. Porque el discurso se conforma de tres elementos, el que habla (hoy diríamos el argumentador), de aquello acerca de lo cual se habla (el tema) y de aquél a quien se dirige, el o lo oyente/s (el auditorio) (Racionero, 2018). Esto como una analogía del rol que cumple un docente en su práctica tradicional, adquiere una connotación especial en las nuevas prácticas que desarrolla un docente en la modalidad virtual o un tutor virtual.

Por otro lado, ideas como las de Kurt Lewin, respecto a "la comunicación de grupos y el efecto de las presiones de grupo, normas de grupo y papeles de grupo sobre el comportamiento y actitudes de sus miembros", siguen vigentes en la comunicación intragrupal y el efecto que tiene la interacción de los miembros en ellos mismos (Macías, 2003). Esto se visibiliza también en la modalidad virtual, en las interacciones de los grupos por medio de comentarios y discusiones respecto a temáticas cercanas al silabo 0 rompiendo estos parámetros en los espacios de interacción virtual.

Es así, que el E- learning, o aprendizaje electrónico, demanda que la interacción se realice a través de comunicaciones eficaces, que aseguren la permanencia de los estudiantes, por ello consideramos oportuna la idea respecto al factor humano y sus efectos no muy positivos en esta esta modalidad, cuando se tergiversa el rol mediador del profesor en el aprendizaje, por la sustitución de la tecnología, olvidando que ésta ha de ponerse al servicio del docente, que es quien habrá de seguir desempeñando el principal papel mediador en el aprendizaje"(Seoane, García, 2008).

Dicho esto, el docente es clave en el proceso de incorporación de las Tecnologías de la Información y Comunicación (TIC) a la enseñanza, para que la acción docente vuelva a ser el núcleo metodológico y organizativo de la integración de las TIC en las universidades. Referido a un rol transformador, convirtiéndose en dinamizador, diseñador de contenidos en nuevos contextos formativos, facilitadores del aprendizaje, orientadores, guías, motivadores, moderadores, tutores virtuales, evaluadores, etc.(Sangrà y González, 2010).

En este marco, es importante precisar que el objetivo de la educación virtual es responder a la gran demanda que se presenta en cada uno de los países del mundo, abrir posibilidades formativas a miles de personas impedidas por diversos factores, facilitar estrategias de educación permanente para la población adulta que quiere iniciar o continuar estudios superiores, acercar los procesos educativos a los procesos reales de la vida.

Así también, cabe precisar que esta modalidad demanda que el estudiante construya su propio aprendizaje y se promueva una autoevaluación permanente, llevando al estudiante a desarrollar la metacognición, esencial para su propio monitoreo. En otros términos "aprender a aprender".(Betancourt, 2010). 
Por otro lado, el maestro adquiere una funciona tutorial, considerada uno de los pilares sobre los que se consolida la educación on-line. La misma que consiste en la relación orientadora de uno o varios docentes respecto de cada alumno en orden a la comprensión de los contenidos, la interpretación de las descripciones procedimentales, el momento y la forma adecuados para la realización de trabajos, ejercicios o autoevaluaciones, y en general para la aclaración puntual y personalizada de cualquier tipo de duda (Padula, 2002), (Berrocoso y Arroyo, 2005).

En esa misma línea, Lugo (2003) sostiene la idea de guía es la que aparece con más constancia en la definición de la tarea del tutor. En la modalidad on-line esta actividad se realiza a través del acompañamiento, la información y el asesoramiento. Es un rol que elabora estrategias adecuadas a las necesidades particulares de los destinatarios.

El ejercicio del rol del tutor on-line se centra en el aprendizaje y no en la enseñanza, por esto su figura no puede construirse como experto transmisor de contenidos, sino más bien como animador y vehiculizador del aprendizaje autónomo que realizan los alumnos. Estas ideas nos llevan a reflexionar sobre un rol más humano, en un entorno tecnologizado más allá del cumplimiento de un silabo y una experiencia curricular, en el sentido, que, si bien la realidad en las formaciones e learning y Blended learning se asumen desde un modelo pedagógico, dentro de los roles, el más cercano al alumno es el del tutor virtual sin perder el rostro humano. (Berrocoso \& Arroyo, 2005).

A decir de Fundesco (1998) el servicio de tutoría a través de la red puede efectuarse mediante las distintas herramientas de comunicación asincrónicas (v.gr. correo electrónico, foro de debate y FAQ) y sincrónicas (v.gr. chat, videoconferencia y pizarra digital) que las plataformas de e-learning suelen incorporar.

Vilà y Rubio (2016). En su estudio sobre Una comunidad de práctica virtual para la cotransferencia. La visión de tutores y tutoras de prácticas del grado de Pedagogía, concluye que esta apuesta formativa demanda cambios en el rol del docente y alumno. Es un ejercicio que tiene sus complejidades, el profesor está acostumbrado a pensar en el contenido no en la actividad, ésta es complementaria. Lograr este cambio en el diseño, transforma la práctica docente, independiente de la modalidad en que se enseñe."

La asunción del triángulo alumno-profesor-contenidos como unidad básica de análisis de los procesos de enseñanza y aprendizaje en contextos virtuales comporta, al mismo tiempo, considerar la articulación entre las actuaciones de profesor y alumnos en torno al contenido y tareas de enseñanza y aprendizaje, la "actividad conjunta" o "inter-actividad" como factor explicativo fundamental del aprendizaje en estos contextos y de su calidad (Onrubia, 2005).

El desarrollo de la tecnología tuvo inicialmente énfasis en el correo electrónico, siendo en algún momento el medio más generalizado para realizar tutorías a través de Internet. En la actualidad los foros de naturaleza asincrónica (Otra herramienta utilizada en los en los LMS), son utilizados extensamente. Sin embargo, esta experiencia evolucionó a la experiencia sincrónica a través de las videoconferencias, el chat, etc. Permitiendo un contacto de reconocimiento personal con seres humano al otro lado del monitor. Estas interacciones en proceso formativos a distancia son abordadas por los alumnos con motivación, la misma que de manera inicial suele ser elevada respecto a la materia y a la metodología. Tal cual se va desarrollando el curso, esta motivación puede sufrir variaciones en función de una gran cantidad de factores académicos, administrativos y personales (Berrocoso y Arroyo, 2005).

Klug y Soledad (2019) plantean que la tutoría se entiende como: "una construcción pedagógica que posibilita, entre otras dinámicas, estimular los procesos de enseñanza aprendizaje y amenizar la vida universitaria" (p.3). En este sentido responde a aspecto técnicos pedagógicos, debe ser diseñada, planificada, coordinada y evaluada para lograr el efecto deseado. 
Como se ha podido dar cuenta, existe abundante información respecto a lo que es o debería ser la función del tutor virtual soportada por Sistemas de Gestión de Información, sin embargo, es claro que el tránsito de prácticas pedagógicas tradicionales respecto a nuevas situaciones de aprendizaje, en formaciones académicas en adultos ha generado tensiones y relaciones de conflicto entre asesor y asesorado, en los foros de interacción en ambientes de aprendizaje a distancia , La capacidad empática de los asesores influencia de forma positiva en la percepción de los tesistas en los foros de asesoría, y la ausencia, de forma negativa generando sentimientos de angustia, desesperación e ira no manifestadas de forma clara y explícita en los foros, tal como lo expresa (Abovsky, Alfaro, \& Ramíres, 2011). El tutor debe valorar el afecto alcanzar y sensibilizar al alumno de distancia (Tenório, Araújo Costa y Tenório, 2016).

En esta misma línea (Cueva, Molerio y Ramirez, 2019) afirman que la acción tutorial puede realizarse de modo vertical, asumiendo el tutor una posición de superioridad con relación a la posición que asume el alumno, o bien de modo horizontal, entendiendo en este caso que la relación entre ambos asimétrica.

En este marco, la naturaleza del presente estudio se realizó, teniendo como objeto de estudio las tutorías virtuales en la Especialización Gestión del E-Learning y Docencia Virtual a través de un sistema de Gestión del Aprendizaje (LMS), Moodle, en el cual se desarrollad el sistema modular de la especialización, gestionada por la unidad de Estudios de Segunda especialidad de la Universidad nacional Pedro Ruíz Gallo (Facultad de Educación).

Las comunicaciones ejecutadas entre docente y estudiantes profesionales tuvieron como objetivo el desarrollo de asesorías para la construcción del trabajo que les permitirá sustentar la especialización en cuestión, en el marco de lo que se denominada Taller de sistematización de Informe Técnico Profesional. En este sentido son dos líneas directrices que abordó el presente estudio, uno relacionado al aspecto tutorial y otro al aspecto tecnológico, en donde ambos convergen en lo que es la Tutoría virtual empática a través de Webinar.

En los últimos años los procesos de asesoría virtuales se vienen desarrollando en nuevas propuestas tecnológicas, denominadas Conferencias virtuales-Webinar". Un Webinario o seminario web es una presentación, seminario, conferencia o taller transmitido a través de Internet. Esta tecnología emergente se está volviendo cada vez más popular debido a su conveniencia y asequibilidad. Sin embargo, se han realizado pocas investigaciones sobre las mejores prácticas para un seminario web interactivo que involucra a los estudiantes en un desarrollo profesional o entorno académico.

Algunas de las Ventajas de hacer un Webinar es que pueda realizarse desde casa. Ahorro en Costes, numerosas Prestaciones. Con los diferentes tipos de Software que permiten realizar esto puedes hacer diferentes tipos de acciones: Compartir tu pantalla con los asistentes, gestionar permisos para que los asistentes puedan chatear entre ellos 0 , todo lo contrario, para que nadie vea quien más está en el Vídeo-Seminario, permitir ronda de preguntas, compartir tu cara a la vez que tu pantalla, etc.

El otro aspecto considerado clave es la tutoría empática, que a través de relaciones socio afectivas, sin generar dependencia o paternalismo, den lugar a situaciones subjetivas y emocionales de confianza y seguridad en los asesorados, evitándose así las frustraciones y deserciones. 


\section{METODO}

El paradigma positivista orientó el estudio, asumido desde el método cuantitativo, se enmarcó en un diseño pre-experimental, de un solo grupo con pretest y postest, se aplicó un cuestionario para conocer las apreciaciones de los estudiantes, a partir de las acciones de tutoría antes de la intervención y después de la intervención. Se hizo uso de la estadística descriptiva para el procesamiento de la información. Planteamiento de Hernández, et al. (2014).

El estudio responde a las siguientes preguntas de investigación: ¿qué características debe tener la tutoría virtual a través de la técnica de webinar en el desarrollo de los talleres de sistematización?

Se consideró como Objetivos General, Implementar la tutoría virtual a través de del software Go to Webinar en estudiantes del Taller de sistematización de la especialidad de Gestión del e-Learning y Docencia Virtual de la Universidad Nacional Pedro Ruíz Gallo. Siendo los objetivos específicos identificar las características de los participantes en la formación de especialización, analizar la percepción de los estudiantes respecto a la tutoría virtual durante las experiencias curriculares, diseñar el Plan de tutoría virtual empática para webinars, ejecutar el plan de tutoría virtual empática y un último objetivo fue Identificar la percepción de los estudiantes después de la ejecución de los Webinar.

\section{RESULTADOS}

Objetivo $\mathrm{N}^{\circ} 01$ : identificar las características de los participantes en la formación de especialización, analizar la percepción de los estudiantes respecto a la tutoría virtual durante las experiencias curriculares

Se identificó cuantas personas por género existen

Tabla 01:

Principales características del grupo de estudio

\begin{tabular}{|c|c|c|}
\hline Ítems & $\begin{array}{c}\mathrm{N}^{\circ} \mathrm{de} \\
\text { encuestados }\end{array}$ & porcentaje \\
\hline más de 10 años de servicio & 15 & $53.57 \%$ \\
\hline menos de 10 años de servicio & 13 & $46.43 \%$ \\
\hline nombrado & 13 & $46.43 \%$ \\
\hline contratado & 15 & $53.57 \%$ \\
\hline más de 30 años de edad & 20 & $71.43 \%$ \\
\hline menos de 30 años de edad & 15 & $53.57 \%$ \\
\hline llevo cursos en Perú Educa & 4 & $14.29 \%$ \\
\hline conocimientos de computación & 13 & $46.43 \%$ \\
\hline capacitación en TIC & 16 & $57.14 \%$ \\
\hline confianza en la modalidad & 14 & $50.00 \%$ \\
\hline TOTAL & 28 & \\
\hline
\end{tabular}

Fuente: Pretest

Nota: 28 encuestados el mayor porcentaje 53.57\% cuentan con más de 10 años de servicio.

$13,46,43 \%$ son nombrados, 4 docentes han desarrollado curso en el portal Perú Educa. 
Los participantes cuentan con conocimientos de computación y experiencia de cursos virtuales.

Figura 01: Principales características del grupo de estudio

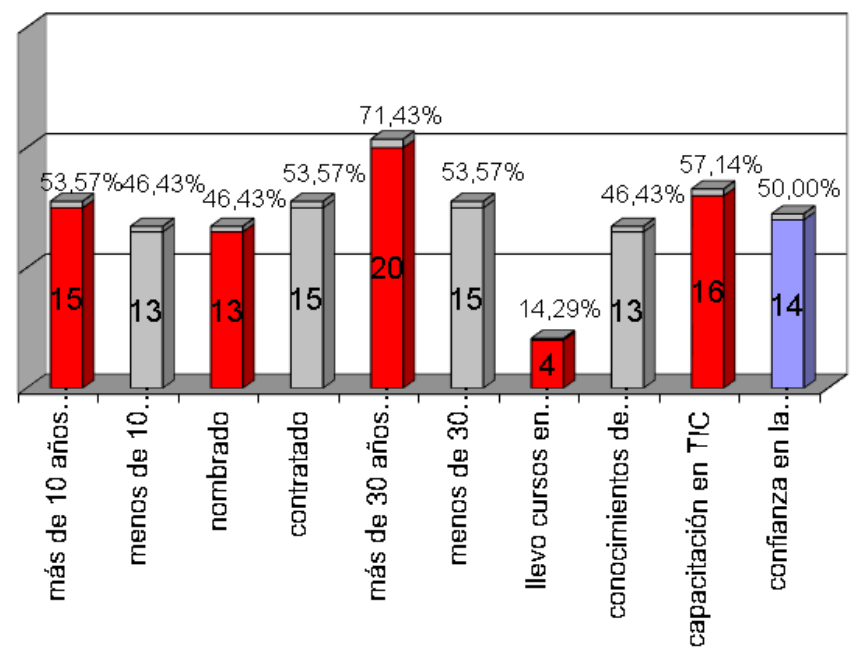

\begin{tabular}{|c|c|c|c|}
\hline Alternativas & \# Encuestas & $\begin{array}{c}\% \\
\text { Respuestas }\end{array}$ & Tahla 02. \\
\hline hombres & 13 & $46.43 \%$ & Datos de \\
\hline mujeres & 15 & $53.57 \%$ & identificación \\
\hline TOTAL & 28 & $100.00 \%$ & por sexo \\
\hline
\end{tabular}

Fuente: Pretest

Nota: identificación por sexo

Figura 02: Datos de Identificación por sexo

\section{Datos de identificación por sexo}

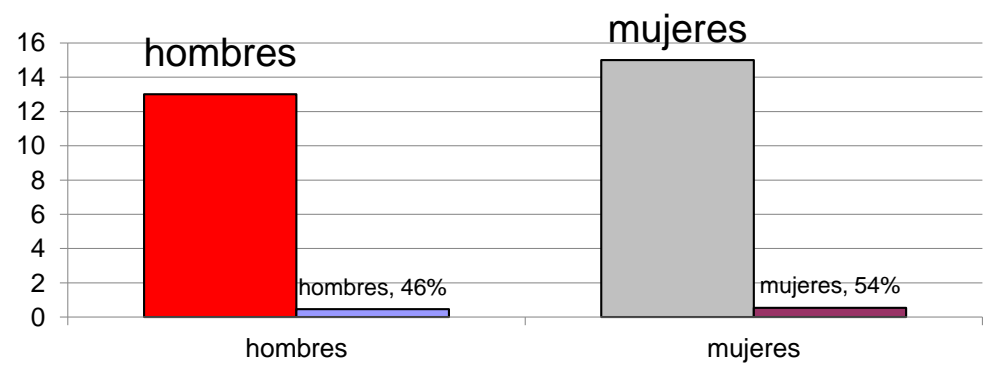

\section{Nota:}

Los resultados nos muestran que el mayor porcentaje de participantes es de mujeres $53,6 \%$ y $46,4 \%$ corresponde a hombres.

Objetivos 01: Identificar la percepción de los estudiantes respecto a la tutoría en su experiencia curricular denominada Taller de sistematización. 
Tabla 03:

Experiencia antes de Webinar para la tutoría virtual

Alternativas

PRETEST

POSTEST

\begin{tabular}{cccccc}
\hline$\#$ & Total & $\%$ & $\#$ & Total & $\%$ \\
Encuestado & Respuesta & & Encuestado & Respuesta
\end{tabular}

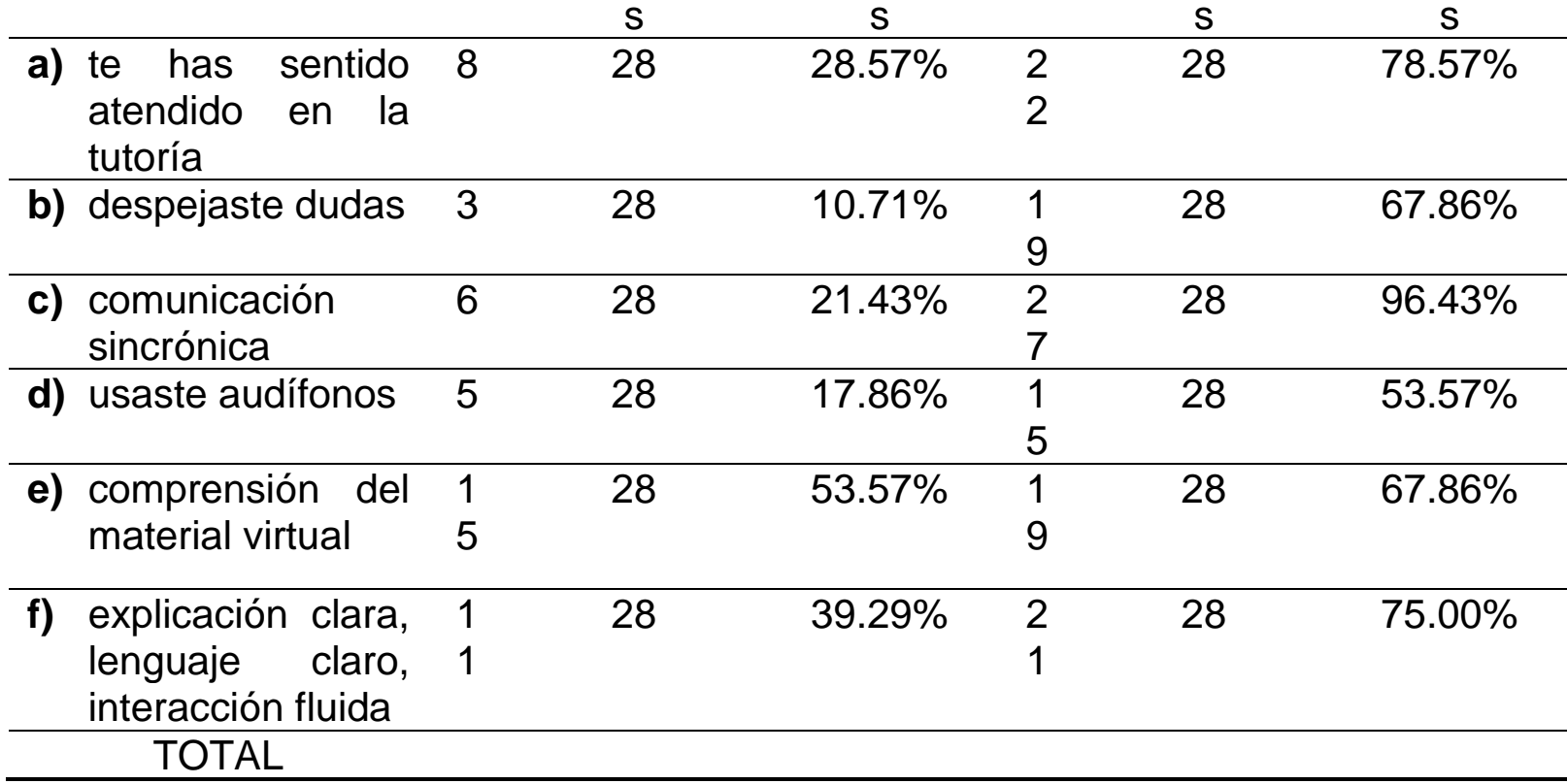

Fuente: pretest y postest a estudiantes de la especialización

Nota: Se recogió información a preguntas cerradas

Se muestra un paralelo del pretest y postest respecto a experiencia previa en webinar.

Se evidencia que los niveles de las percepciones aumentaron se elevaron respecto a la experiencia inicial de los estudiantes.

Los participantes dan cuenta que tiene un conocimiento previo respecto a la compresión de materiales.

Figura 02: Experiencia antes de Webinar para la tutoría virtual

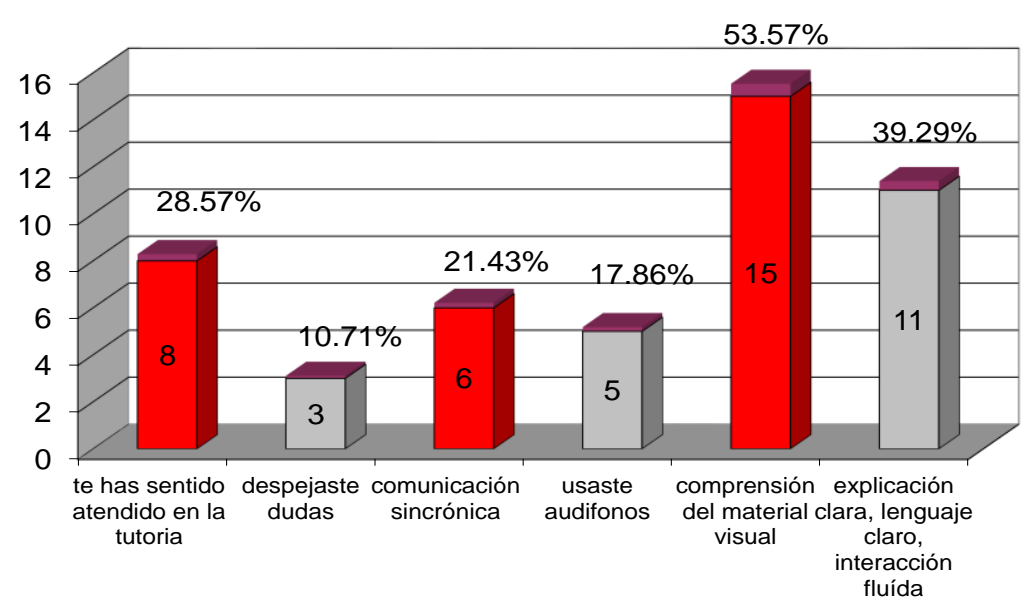

Nota: Se identifica que los estudiantes manifiestan poca atención en la tutoría $28,57 \%$.

En términos generales se encuentran muchas deficiencias en la tutoría virtual. 
Figura 03: Experiencia de tutoría virtual después Webinar.

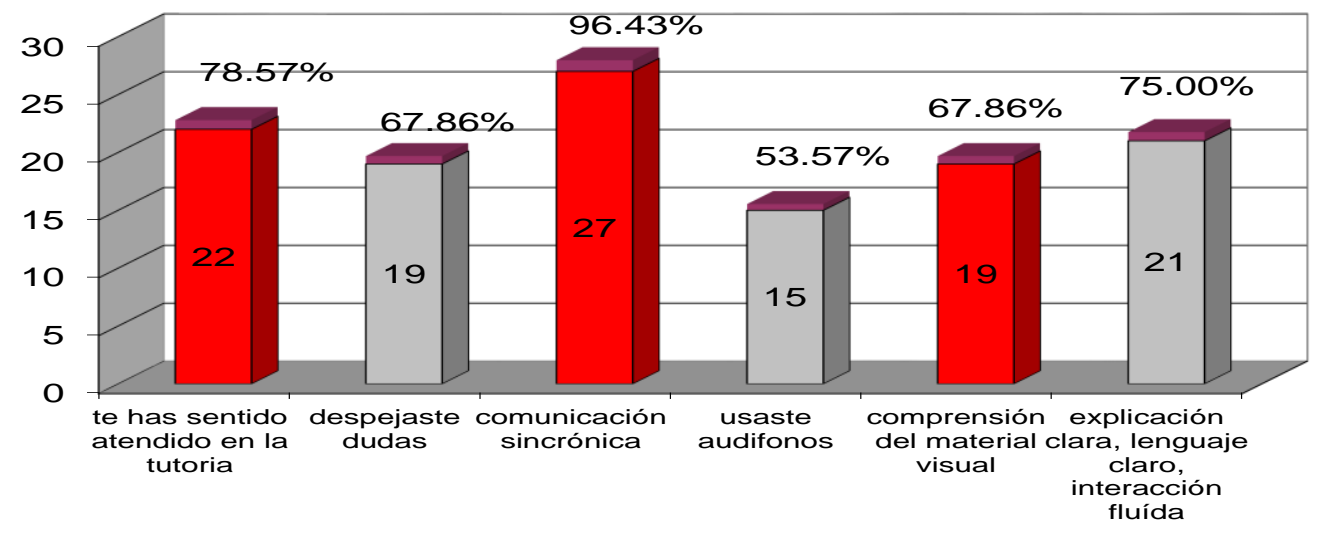

Fuente: postest a estudiantes de la especialización

Nota: Se identifica que hubo incremento respecto a la evaluación inicial

En términos generales se identifica que se mejoró la experiencia en cuanto a la tutoría virtual.

\section{Características de la intervención tecnológica:}

A partir de la limitaciones identificadas se diseñan un plan de tutoría utilizando la técnica Webinar, utilizado para ello la versión de prueba de Go to Webinar plataformas que ofreció una serie de herramientas, que permitieron cubrir las deficiencias desde el aspecto tecnológico para la comunicación sincrónica, se insistió en el uso del micro porque este facilita la comunicación verbal en tiempo real, articulado a este aspecto, se reconfiguró la función del tutor virtual, desde tutor para fortalecer la empatía, teniendo en cuenta aspectos claves como un discurso con ideas claras, con mensaje e instrucciones en leguaje preciso para una comprensión eficaz de los contenidos, se introdujo un espacio previo al inicio a las sesiones de asesoría para recibir y saludar de manera personal a los estudiantes que se incorporaban a las sesiones, se dosificó el tiempo organizándose las participaciones de cada miembro al que se le identificada verbalizando su nombre, creando un "clima virtual de confianza". Las apreciaciones de estas acciones fueron recogidas a través de preguntas adicionales en el postest.

Objetivo 02: Diseñar el Plan de Trabajo para desarrollar la tutoría virtual en el taller de sistematización.

La tutora virtual basó el cumplimiento de este objetivo en el diseño e implementación de los elementos del Plan de trabajo para la tutoría virtual 
Plan de tutoría virtual para taller de sistematización en la especialidad gestión del eelearning y docencia virtual

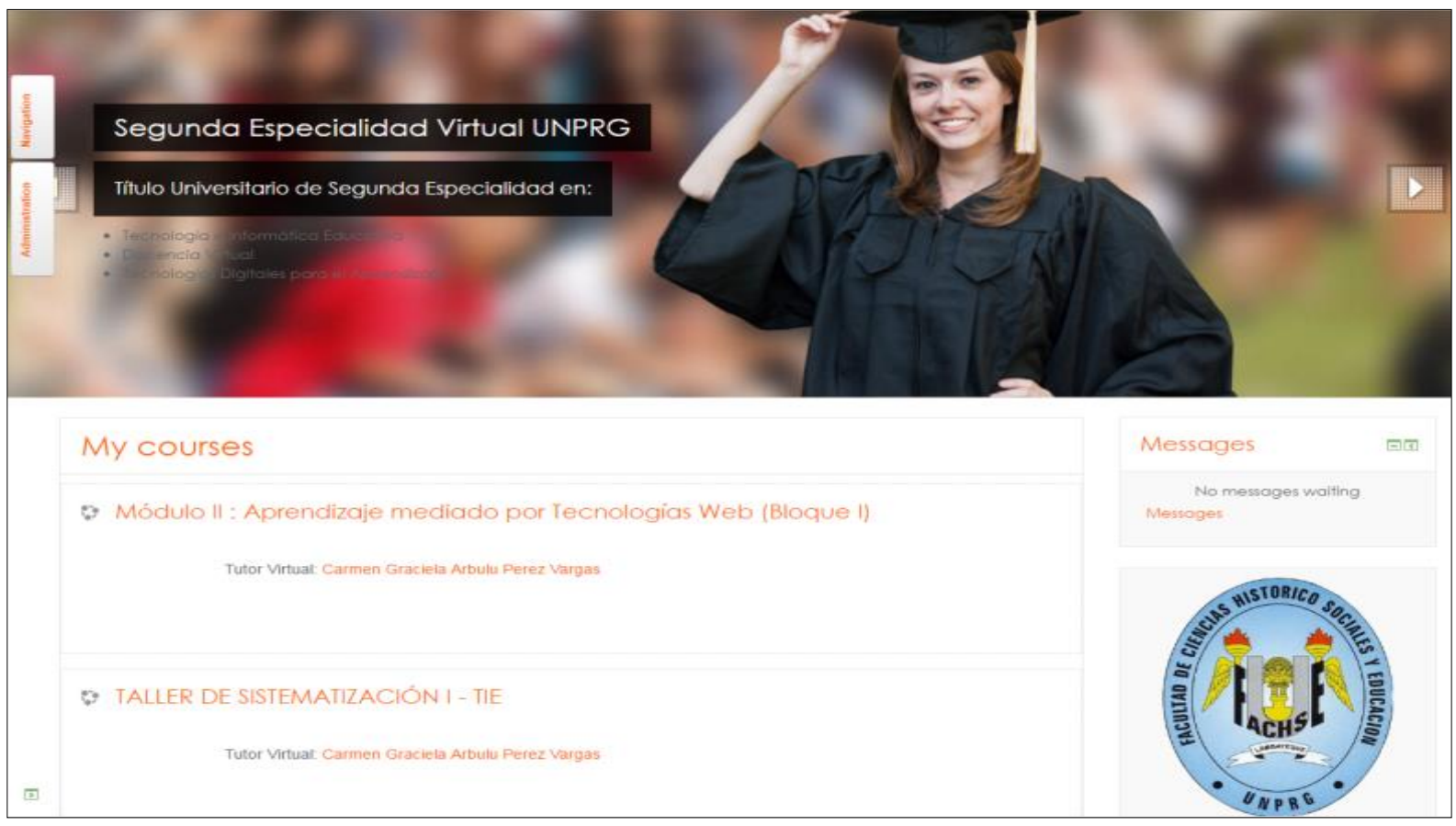

El abordaje conceptual de la experiencia curricular parte de la consideración de las bases tecnológicas sobre las que se construyen los entornos virtuales y la utilización de herramientas para la gestión de la comunicación como parte de la tutoría virtual, ofrecidas por el software utilizado, Go to Webinar.

\section{Acciones para la programación del Webinar}

- Registro del Webinar para darlo a conocer a los estudiantes.

- Configuración de la fecha establecida para la difusión de la dirección del seminario Online para que los estudiantes acepten y se registre.

- Difusión de la dirección del webinar en el aula virtual.

- Estructuración de la tutoría de la siguiente manera: Presentación, contenido de valor (discurso empático), se comenta aspectos positivos del taller virtual y los beneficios de la modalidad que se ofrece.

Por otro lado, el enfoque humano, asumido por el tutor, desde un discurso empático, adaptando cuatro funciones principales: a. Informa al estudiante la competencia del programa y el plan de trabajo con la finalidad de apoyar su desempeño académico. b. Retroalimenta al alumno acerca de su progreso y logro de los objetivos y sugerencias para mejorar su rendimiento escolar. c. Apoya en el desarrollo de soluciones técnicas, dudas y recomendaciones para realizar trámites administrativos $d$. Comunica mediante las herramientas de la plataforma educativa las acciones tutoriales para el acompañamiento de los estudiantes (Arranz, 2008). Todas estas acciones con empatía y tolerancia, valorando la diversidad cultural que cada estudiante.

La descripción del abordaje conceptual del Taller de Sistematización es una adaptación del abordaje conceptual en la modalidad presencial, lo cual fue motivo de la presente intervención.

La naturaleza del taller de sistematización tiene dos momentos: plenaria y atención personalizada. La plenaria está destinada a las orientaciones de carácter genérico que debe brindar el docente, válidas para todos los asistentes al margen 
del informe modular específico presentado. Usualmente se centran en la estructuración u ordenamiento general del informe. La atención personalizada implica un asesoramiento directo y sincrónico en el entorno del Webinar.

Se observa que las valoraciones hechas por los estudiantes se han elevado, siendo la más alta a que se reconoce que existe una comunicación sincrónica $96,43 \%$, esto significa que el nivel de comunicación se ha mejorado. Otro aspecto que se valora en gran medida es la explicación que hace el tutor, con un lenguaje claro, e interacción fluida, esto tiene que ver con la empatía puesta en práctica y calidad en el discurso, favorecido por la tecnología, ambos aspectos se incorporan en el Webinar.

Figura 04: Elementos de la tutoría virtual empática a través de webinar en talleres de sistematización

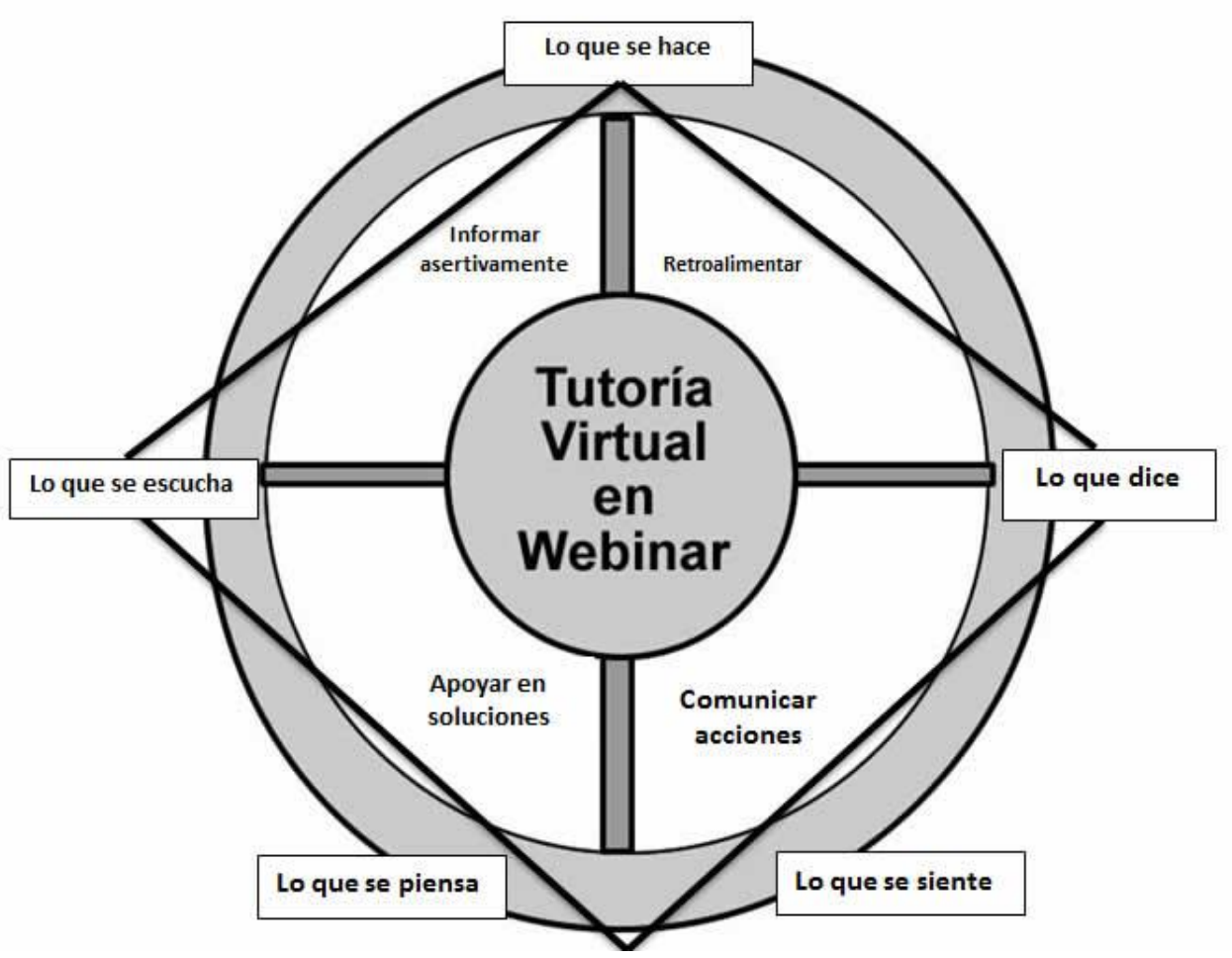

Fuente: Elaboración propia

\section{CONCLUSIONES}

Se identificó en la evaluación inicial que el 53,6\% son de sexo femenino y el $46.4 \%$ sexo masculino, además los mismos expresan desconformidades respecto a su atención en la tutoría, el $28.57 \%$ se sintieron atendidos en sus experiencias con tutoría virtual, el $10.71 \%$, expresa que despejaron dudas, el $21.43 \%$ participó de comunicación asincrónica, el $17.86 \%$ uso audífonos, estos permiten escuchar y hablar, el no utilizarlos limitan la calidad de la comunicación, el 53.57\% comprende el material de as experiencias curriculares, el 39.29\%, reconocen explicación clara, lenguaje claro, interacción fluida.

El plan diseñado para mejorar la tutoría virtual utilizando los webinar como técnica para la asesoría en el Taller de Sistematización de la Especialidad de Gestión del e-Learning y Docencia Virtual se basó en cuatro elementos Información, Retroalimentación, Apoyo en soluciones técnicas, dudas, Comunicación de acciones mediante las herramientas del Software para Webinar y el aula Virtual, sostenidos 
en la comunicación empática del tutor, manejando tolerancia, paciencia, respeto a la interculturalidad desde lo que se escucha, lo se piensa, lo que se dice, lo que hace y lo que se siente.

Después de ejecutado el Webinar, se recogió información respecto a la percepción de los estudiantes las mismas que dieron cuenta que los niveles de satisfacción aumentaron, estos resultados nos muestran una apertura por parte de tutor virtual y estudiantes ante nuevas formas de comunicación y metodologías en proceso formativos. Los Webinar y la metodología para su implementación demandan en los estudiantes resistencia inicial, seguida de adaptación y finalmente aceptación y acomodación. Estos procesos permitieron que los estudiantes logren entregar su producto acreditable, que fue el informe modular, bajo los lineamientos de la Unidad de Estudios de Segunda Especialidad.

\section{REFERENCIAS}

Abovsky, A., Alfaro Rivera, J. A., \& Ramíres Montoya, M. S. (2011). Relaciones interpersonales virtuales y empatía en el manejo de conflictos personales: ¿Factores determinantes en el proceso de asesoría y en la formación de investigadores educativos a distancia?

Almeida, A. R. de, Silva, C. L. da, \& Santoyo, A. H. (2018). El Tutor en los entornos virtuales de Aprendizaje. Universidad y Sociedad, 10(3), 134-141. https://doi.org/10.3182/20120611-3-IE-4029.00015

Berrocoso, J. V., \& Arroyo, M. del C. G. (2005). La funcion tutorial en entornos virtuales de aprendizaje: comunicación y comunidad. Revista Latinoamericana de Tecnología Educativa - RELATEC, 4(1), 153-167.

Betancourt, A. M. (2010). Educacion a Distancia Y La Funcion Tutorial. Unesco, Dos, 1-149.

Cueva, Jorge; Molerio, Liliana; Ramirez, M. (2019). Las tutorías virtuales en el proceso universitario. 11, 260-268.

Espinoza Freira, E. E. (2018). El tutor en los entornos virtuales de aprendizaje. Universidad y Sociedad, 10(1), 201-210. Recuperado de http://rus.ucf.edu.cu/index.php/rus

Macías, G. J. (2003). Teorías de la comunicación grupal en la toma de decisiones: contexto y caracterización. TDX (Tesis Doctorals En Xarxa), 325. Retrieved from http://www.tdx.cat/handle/10803/4112\%0Ahttp://www.tdx.cat/bitstream/handle/10803/4 112/gjmc1de1. pdf;jsessionid=9BFCF3E1DBBFB6A0886B7B3A2EA0B8CB? sequence= 1

Onrubia, J. (2005). Aprender y enseñar en entornos virtuales: actividad conjunta, ayuda pedagógica y construcción del conocimiento. RED. Revista de Educación a Distancia, 1-16. https://doi.org/10.6018/red/50/3

Racionero, Q. (2018). Retórica. In GREDOS.S.A (Ed.), BIBLIOTECA CLÁSICA GREDOS, 142 (Primera). https://doi.org/10.14195/978-989-26-1596-7_20

Sangrà, A., \& González-Sanmamed, M. (2010). The role of information and communication technologies in improving teaching and learning processes in primary and secondary schools. ALT-J, 18(3), 207-220. https://doi.org/10.1080/09687769.2010.529108

Seoane, A M; García, F. J. (2008). La función tutorial desde la Antigüedad hasta nuestros días: fundamentos filosóficos y modelos clásicos de monitorización para su aplicación a contextos de E-learning. Comunidad de Aprendizaje En Red. Los Retos Para Consolidarla, 103-124.

Tenório, A., Araújo Costa, M. de F., \& Tenório, T. (2016). A influência da empatia como competência socioafetiva na atuação de tutores a distância. Revista Brasileira de Aprendizagem Aberta e a Distância, 15. https://doi.org/10.17143/rbaad.v15i0.270

Vilà, Ruth; Rubio, M. J. R. (2016). Una comunidad de práctica virtual para la cotransferencia. La visión de tutores y tutoras de prácticas del grado de Pedagogía. 1727. 